\title{
CORRIGENDUM
}

\section{The putative tumor suppressor RASSF1A homodimerizes and heterodimerizes with the Ras-GTP binding protein Nore1}

Sara Ortiz-Vega, Andrei Khokhlatchev, Maria Nedwidek, Xian-feng Zhang, Reinhard Dammann, Gerd P Pfeifer and Joseph Avruch

DOI: $10.1038 /$ sj/onc/1205378

Correction to: Oncogene (2002) 21, 1381-1390. DOI: $10.1038 / \mathrm{sj} /$ onc/1205192

The authors wish to apologise for an error found in the discussion section. A revised version of the text is given below:

Moreover, although Nore binds a non-prenylated $\mathrm{Ki}$ RasG12V slightly better than Ha-RasG12V in a twohybrid assay, wildtype cHa-Ras binds Nore better than cKi-Ras during transient expression in COS cells. 